\title{
Opinion
}

\section{The perspectives and the challenges of Paediatric Radiology: An interview with Dr Georgia Papaioannou, Head of the Paediatric Radiology Department at the 'Mitera' Children's Hospital in Athens, Greece}

\author{
IOANNIS N. MAMMAS ${ }^{1-3}$ and DEMETRIOS A. SPANDIDOS ${ }^{1}$ \\ ${ }^{1}$ Laboratory of Clinical Virology, School of Medicine, University of Crete, 71003 Heraklion; ${ }^{2}$ First Department of Paediatrics, \\ University of Athens School of Medicine, 11527 Athens; ${ }^{3}$ Paediatric Clinic, Aliveri, 34500 Island of Euboea, Greece
}

Received July 22, 2019; Accepted August 26, 2019

DOI: $10.3892 /$ etm.2019.7949

\begin{abstract}
Dr Georgia Papaioannou, MD, PhD, Head of the Paediatric Radiology Department at 'Mitera' Children's Hospital in Athens (Greece), is one of the most talented Paediatric Radiologists in Greece. She graduated from the Medical School of Athens of the National and Kapodistrian University of Athens in 1996 and she was trained in Radiology at the 2nd Department of Radiology of the Medical School of Athens and in Paediatric Radiology in Great Ormond Street Hospital for Children (GOSH) in London (UK). Her special interests focus on foetal magnetic resonance imaging (MRI) and special applications of paediatric MRI, such as MR enterography and MR urography. According to Dr Papaioannou, medical education in Paediatric Radiology has to be of excellent quality, raising professional career and academic opportunities. Paediatric Radiology focuses on the diagnostic imaging of foetuses, neonates, infants, children and adolescents and represents the oldest subspecialty of Radiology. The advent in technology and computer science, which is strongly associated with Radiology, has launched a new era for Paediatric Radiology training and practice. Digital imaging and archiving, telemedicine, fused imaging, evolution of sonographic equipment, computed tomography (CT) and MRI scanners have opened new horizons to the understating of paediatric physiology and pathophysiology and have contributed significantly in precision Medicine and the implication of targeted therapies into clinical practice. Modern paediatric imaging focuses on radiation protection issues and launches faster sequences in all modalities, in order to reduce the number and duration of sedation. The diagnosis of neonatal and paediatric viral infections is routinely clinical
\end{abstract}

Correspondence to: Professor Demetrios A. Spandidos, Laboratory of Clinical Virology, School of Medicine, University of Crete, 71003 Heraklion, Greece

E-mail: spandidos@spandidos.gr

Key words: Paediatric Radiology, Paediatric Virology, medical education, radiology protection, MRI, Georgia Papaioannou and laboratory; however, Paediatric Radiology assists the diagnostic approach by excluding other entities, if that is required, i.e., in complex cases or hospitalized children, and mainly by revealing associated complications.

\section{Contents}

1. Introduction

2. Questions and Answers

\section{Introduction}

Dr Georgia Papaioannou, MD, PhD (Fig. 1), Head of the Paediatric Radiology Department at the 'Mitera' Children's Hospital in Athens (Greece), is one of the most talented Paediatric Radiologists in Greece. She graduated with honours from the Medical School of Athens of the National and Kapodistrian University of Athens in 1996. She was trained in Radiology working as a Radiology resident at the 2nd Department of Radiology of the Medical School of Athens. Following successful examination, she attended a Fellowship in Paediatric Radiology in Great Ormond Street Hospital for Children (GOSH) in London (United Kingdom) from 2004 to 2005. In 2004, she completed her PhD in Medical School of Athens; the title of her thesis was 'Metallic stents. Application in the treatment of iliac artery atherosclerotic disease' (1).

Dr Papaioannou has worked as a Scientific Associate in the 2nd Department of Radiology of Medical School of Athens (2001-2004), Consultant Paediatric Radiologist in Royal London Hospital NHS Trust, London (2005) and Consultant Radiologist in the Greek National Health System at the 2nd Department of Radiology of the Medical School of Athens in 'Attikon' University Hospital (2007). She has been working in 'Mitera' Children's Hospital since 2009 and has been assigned as Director of Paediatric Imaging in 2011. She has a special interest in foetal magnetic resonance imaging (MRI) and special applications of paediatric MRI (MR enterography, MR urography). She has been granted several awards, among which 
the 'outstanding educational poster Award' from the World Federation of Pediatric Imaging (WFPI) in the International Pediatric Radiology (IPR) meeting at Chicago (USA) in May, 2016. Recently, in the 2018 European Congress of Radiology (ECR) in Vienna (Austria), she was awarded with the most viewed scientific exhibit, entitled 'Placental Imaging in a High-filed open MRI: Practicalities and initial findings'.

Dr Papaioannou has co-authored 4 scientific books on paediatric imaging (2-5), 29 scientific international papers - see selected recent publications in the References section (6-11) - and 4 electronic papers (12-15). She is member of several scientific societies, including the European Society of Pediatric Radiology (ESPR) and reviewer in a significant number of international scientific journals in Radiology, Paediatric Radiology and Paediatrics. She has been member of the organizing committee in the ESPR's congress in 2012 and in 7 Paediatric Radiology courses, organized at 'Mitera' Children's Hospital. She has been invited to give a lecture in 8 international meetings and has performed over 110 scientific presentations in national and international meetings.

To date, Dr Papaioannou has actively supported the Paediatric Virology Study Group (PVSG) and has participated with enthusiasm in all 'workshops on Paediatric Virology' since 2016 (16-18). Her plenary lectures have focused on 'multimodality imaging of viral infections in children' (19), 'foetal and neonatal brain imaging in viral infections' (20) and 'radiology issues of influenza in children' (21). In 2016, on October 8th, she co-chaired the '2nd Workshop on Paediatric Virology' and she received the '2016 Paediatric Virology Award in Paediatric Radiology'. In the forthcoming '5th workshop on Paediatric Virology', her plenary lecture will focus on radiology findings of respiratory syncytial virus (RSV) - positive bronchiolitis in children.

\section{Questions and Answers}

Question: Radiological imaging is extremely valuable as a diagnostic tool in the paediatric population and during the last years it represents one of the fastest growing areas in Medicine. What is Paediatric Radiology and what is its history as a separate subspecialty of Radiology?

Answer: Paediatric Radiology is a subspecialty of Radiology, which focuses on diagnostic imaging of foetuses, neonates, infants, children and adolescents. A basic principle in Paediatrics is that children should not be considered as small-sized adults, as they differ in development, physiology and pathology. This axiom is much supported by Paediatric Radiology as well, and major effort is applied in training of paediatric radiologists in dedicated paediatric departments. It could be argued that Paediatric Radiology is the oldest subspecialty of Radiology, since $\mathrm{x}$-rays where produced in children's hospitals back in the early 1900s. Since then the advent of technology has launched new aspects in practicing Radiology subspecialties and Paediatric Radiology in particular. Nowadays, it is impossible to approach any paediatric pathology without the support and scrutinized analysis of a paediatric radiological study. Back in 1945, Dr John Caffey published his textbook on paediatric $\mathrm{X}$-ray diagnosis and since then he has been considered the 'founder' of Paediatric Radiology. The past decades across the Atlantic specific societies focusing on paediatric imaging have been founded, i.e., the Society of Paediatric Radiology (SPR)

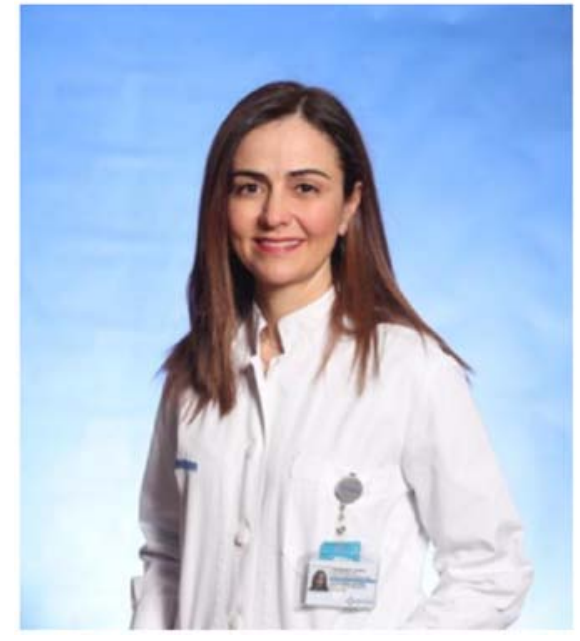

Figure 1. Dr Georgia Papaioannou, MD, PhD, Head of the Paediatric Radiology Department at 'Mitera' Children's Hospital in Athens (Greece) and Chair of the '2nd workshop on Paediatric Virology' held in Athens on October 8th, 2016.

in the US and the European Society of Paediatric Radiology (ESPR), aiming to gather pioneers and experts from all over the world, establish guidelines and exchange opinions on trivial but also rare and demanding paediatric issues, so that consensus of approach and practice can be drawn and applied in different parts of the world by paediatric radiologists.

Question: However, in several countries Paediatric Radiology has not yet been recognized as an official subspecialty. What are the required standards of running a successful training programme in Paediatric Radiology?

Answer: Unfortunately, this is the case in Greece as well. However, several Greek radiologists devote their practice in paediatric hospitals and many of them have obtained a Paediatric Radiology fellowship and experience in paediatric hospitals across the Europe and the US. Obviously, education in Paediatric Radiology has to be of high standard, with the potential of professional career and academic opportunities. However, even nowadays many countries do not provide a dedicated training for Paediatric Radiology. Organizing a training programme in Paediatric Radiology can be very challenging and demanding as it has to coordinate a steep training schedule according to the state-of-the-art approaches, it should be updated when this is required, use multimodality rotation and multidisciplinary medical communities, ideally in busy national children's hospitals with the potential of international exchange of staff and international affiliations. It has to provide an international communication channel, where each society will create an educational pool of teaching files and a platform to exchange knowledge, but also express arguments. It should also align to an ideal plan of continuing professional education, providing financial support opportunities to the younger members and liaise with international authorities. Ideally, it should provide the opportunity of exchanging posts, dedicated either to scientific or clinical fellowship in several paediatric referral centres.

Question: Are currently Radiology residents encouraged to choose Paediatric Radiology as their preferred career choice? 
Answer: Unfortunately, there is a global lack of paediatric radiologists and residents do not include this subspecialty in their top choices. The European Paediatric Radiology Community has launched several educational courses, which aim to present multiple Paediatric Radiology topics to Radiology residents and young doctors. Additionally, significant and robust Radiology meetings, such as the annual meeting by the Radiological Society of North America (RSNA) and the ECR, had special focus in Paediatric Radiology the past decade. In educational and referral hospitals, the teaching programme for residents highlights among other subspecialties the individuality and uniqueness of Paediatric Radiology. Educational resources of the Paediatric Radiology societies promote online education with atlases, interactive ibooks, educational material, calendar of events, learning platforms, where residents have the opportunity to personalize their education but also exchange opinions with peers. Finally, Radiology communities launch scholarships in referral paediatric hospitals for fellowships in Paediatric Radiology.

Question: Diagnostic or interventional Paediatric Radiology? Which one do you prefer?

Answer: I personally find diagnostic Paediatric Radiology very interesting and challenging; I have the chance to get involved with different modalities, several systems and pathologies and the whole spectrum of ages, ranging from foetuses to young adults. In certain modalities, i.e., ultrasound, the personal communication and interaction with the young patient makes my practice a daily pleasure, through the process of earning their trust and getting them to cooperate! A smiley reward is a unique experience in my practice! Interventional Radiology is a valuable tool for clinicians, as it is usually problem solving, diagnostically and therapeutically.

Question: Excellence in paediatric imaging, paediatric image-guided interventions, radiation protection, evidence-based Medicine, precision Medicine, progress in technology methodology, foetal and neonatal imaging. What are the most significant advances in Paediatric Radiology of the last ten years?

Answer: During the past decade, there have been several advances in Medicine as well as Radiology. The advent in technology and computer science, which is strongly associated with Radiology, has launched a new era for Paediatric Radiology practice. Digital imaging and archiving, telemedicine, fused imaging, evolution of sonographic equipment, computed tomography (CT) and MRI scanners have opened new horizons to our understating of paediatric physiology and pathophysiology. Focusing on radiation protection for the paediatric population, certain studies have been omitted and replaced by advanced imaging, i.e., intravenous (iv) urograms by MR urography. Overall, the introduction of MR into paediatric imaging practice has undoubtedly revolutionized the management of young patients, starting from the foetal age. However, ultrasound has also evolved and imaging has moved to a next era, where any part of the body can be imaged in detail, and new techniques, i.e., elastography, have been introduced. Fused imaging, positron emission tomography (PET)/CT and PET/MR promise a more focused insight to Paediatric Oncology and targeted treatment. Finally, teleradiology is the huge challenge for the near future, as it raises the potential of an expert diagnosis in areas where there is lack of paediatric radiologists, but also the development of paediatric imaging networks between institutions worldwide.

Question: How significant is multi-institutional, national or international collaboration in your field?

Answer: It is of highest importance as it enables exchange of opinions, results and provides a huge scientific data pool where statistical results can be extrapolated in order to aid to scientific advent. A growing body of imaging professionals with focus on improving paediatric imaging is of outmost importance in order to achieve future development. Towards this aim, several Task Force Committees are set and work jointly across the European Paediatric Radiology Community.

Question: As a paediatric radiologist you are a member of several multidisciplinary teams. How difficult is this?

Answer: Participating in a multidisciplinary team requires extra effort and time; however, it is extremely rewarding as it gives me an insight to the clinical scenario, a more complete approach and a better understanding of imaging findings on each case. Needless to highlight that cases are usually not straight forward. Additionally, clinical Radiology, which is accomplished with multidisciplinary teams, is the way forward in my field.

Question: Do you believe that paediatric ultrasound could be included in the regular paediatric examination in the future? Answer: I have to admit that my opinion regarding where Paediatric Radiology stands in the child's examination is rather traditional. History and clinical examination are the corner stones of reaching the correct diagnosis. However, once they are performed, I do believe that the next crucial step is expert paediatric ultrasound, as in several cases it may direct the differential diagnosis.

Question: One of the challenges for paediatric radiologists is to gain the child's trust and co-operation before and throughout the duration of an examination, which can be difficult in children who may be ill or in pain. What is the secret of your outstanding co-operation with children?

Answer: The sick child is usually unwilling to relax in every way. He will either be angry and crying or very reluctant to cooperate. Often, our little patient is more stressed than sick and the clinical signs are confusing. On top of that, we usually encounter stressed and very anxious parents. This makes our job more challenging. My initial approach is to smiley welcome the child with his parents, without expressing any hurry, giving them time to help the child lie on the bed and also provide me with a history. During this time, I try to earn child's trust and the best way of doing that is introducing a toy, according to the child's age, or starting a dialogue regarding the child's school life, in older children and adolescents. Then, I explain to the child the procedure of the examination, using child-friendly terminology. I always ask the parents to get involved in the procedure, either by helping the child with a toy, or by motivating them to talk, sing or cuddle them during the examination. During the scan, I warn the child if an unusual experience is to come, i.e., pressure pain. Finally, at the end of the scan, I am willing to provide a small oral report to the parents. 
Question: To date, several concerns have been raised about the potential harmful effects of radiation as well as of some anaesthetic and radiological agents in children. What are the potential health risks of paediatric imaging? How do you address these risks with parents and relatives?

Answer: Over the past decades, radiation protection issues have been raised and campaigned worldwide, based on the fact that children are more sensitive to radiation damage, acute and accumulative, as their tissues are fast growing and they have larger life expectancy than adults. Nowadays, paediatric radiologists are familiar with the 'As Low As Reasonably Achievable (ALARA)' and Image Gently campaigns and all paediatric departments should modify their protocols according to these guidelines and recommendations, bearing in mind that modern imaging focuses on producing diagnostic imaging of a lesser quality if the radiation dose is kept to 'as low as achievable'. The advent of technology has launched faster sequences in all modalities, so that the number and duration of sedation procedures has been reduced. When anaesthesia is applied, paediatric anaesthetists are informed by paediatric radiologists regarding the aim and the duration of the study, in order to apply the required sedation and are present during the scan to minimize adverse effects.

Question: Excellent quality of medical, specialty and subspecialty education or profession development? How significant is continuing education in Paediatric Radiology?

Answer: Continuing education is of outmost importance for all medical specialists, especially paediatric radiologists, as technology rapidly alters the daily practice. Additionally, advents in treatment create a new reality and only continuing education can reinforce adequate practice.

Question: What are the recent advances in paediatric neuroimaging?

Answer: There has been an advent in MRI techniques over the past decade, which has affected adult neuroimaging and has provided crucial insight into the structural integrity and functional aspects of the developing brain. The introduction of 3T MRI systems has enhanced this field, together with the functional applications, i.e., diffusion tensor imaging, MR spectroscopy, functional MRI, MR perfusion, volumetric imaging, arterial spin labelling and fused MRI. The field of paediatric neuroimaging has been also affected and continues to evolve, with emphasis on high spatial resolution, faster scan times, as well as a quest for visualization of the functional aspects of the human brain. MR vendors are focusing on optimizing MR technology to make it safe and suitable for children, given the fact that their size is much smaller than adults and their CNS development demonstrates inherent neuroanatomical differences. The eventual goal would be to evolve as potential biomarkers for predicting neurodevelopment outcomes and prognostication.

Question: The justification and optimization in the use of CT in children balancing dose and quality is really challenging. How difficult is the development of paediatric CT-protocols? Answer: Currently there are suggested paediatric CT protocols, available from the vendors, according to the type of the scanner and the radiation protection campaigns. However, each department should work with the scanner's applicators and the science physicists on reducing the dose for each examination, according to the area scanned and the body parameters of the child. Our aim is to produce diagnostic images rather than high quality images.

Question: MRI is a revolutionary examination with an increasing usefulness in our paediatric tertiary clinical practice. Which are the most significant clinical implications of MRI in paediatric practice?

Answer: Traditionally, MRI is superb in imaging the brain, the spine and the musculoskeletal system. However, relatively new applications that have gained great clinical acceptance are MR enterorrhaphy, MR urography, whole body MRI and cardiac MRI. These applications focus on providing not only superb anatomical imaging but also valuable functional information, frequently replacing other procedures that have radiation burden, i.e., fluoroscopical enterography, scintigraphic studies and intravenous urography, and cardiac catheterization.

Question: Advances on Paediatric Radiology are based on Radiology for adults. How difficult is techniques and protocols that are designed for adults to be implemented in children?

Answer: The technical challenge in applying modalities and protocols designed for adults to children is both the smaller size of the examined area but also the differences in certain functions in children, i.e., increased heart rate in neonates when scanning the chest and the heart. Technology focuses continuously on launching ultra-fast protocols and techniques on each modality and also on improving the resolution when imaging small sized structures. Obviously, the application of paediatric tailored protocols is more challenging in general imaging departments and hospitals, where children are imaged in the same area as adults.

Question: Paediatric Radiology and Oncology. How difficult is for you when during your practice you come across to an undiagnosed tumour in a foetus, neonate, child or adolescent? What are your feelings when you diagnose a malignant tumour in a child?

Answer: It is really difficult. It not always straight-forward to diagnose whether a new mass is malignant or benign; this is more challenging when the newly diagnosed mass is an incidental finding. In these cases, I try to remain calm during the scan, perform an examination of the highest quality, in order to get a diagnostic baseline imaging, identify the organ of origin, the association of the mass with adjacent structures and suggest further imaging for mapping and staging. It is very unpleasant to inform the parents about the result and this is a feeling that one cannot be acquainted with. Usually, the announcement is done in cooperation with the clinicians; we are lucky to have a very active Paediatric Oncology department in our institution and proper actions are taken without delay.

Question: Radiology plays an important role in the detection and evaluation of physical child abuse. What is your experience in this field?

Answer: During my fellowship in GOSH, I had the opportunity to get expert training in non-accidental injury (NAI). However, 
NAI is a very delicate issue and paediatric radiologists need strong support from other clinicians and social resources in order to make the correct diagnosis and protect the abused child. In Greece, I have come across some cases of NAI; my training has appeared valuable in order to organize an effective reaction with the support of my colleagues.

Question: How vulnerable is a paediatric radiologist in overdiagnosing or misdiagnosing?

Answer: Every radiologist who is isolated from the clinician's feedback and is solely dealing with images is vulnerable to misdiagnosis. This is harder in younger age groups, where cooperation is not guaranteed and signs and symptoms may be vague.

Question: You are considered as one of the most talented, reliable and evidence-based Paediatric Radiology experts in Greece. What is the key of your success?

Answer: Thank you for your kind comments. I was lucky to have been trained in a referral and well-organized department at GOSH, which gave me the fundamentals, the inspiration and motivation to continue and set my practice in Greece according to their high standards. This is enhanced regularly with continuous education through Paediatric Radiology meetings and societies. Albeit the challenges I face on a daily basis, I am very enthusiastic and enchanted with my job and I am lucky enough to practice with a team of people that share the same enthusiasm and operate as a whole.

Question: Paediatric Radiology and Paediatric Virology. What is the role of Paediatric Radiology in the diagnosis of neonatal and paediatric viral infections?

Answer: The diagnosis of neonatal and paediatric viral infections is routinely clinical and laboratory. Paediatric Radiology assists the diagnostic approach by excluding other entities, if that is required, i.e., in complex cases or hospitalized children, and mainly supports their best treatment in case of complications. Rarely, it may suggest the diagnosis, i.e., findings of acute necrotizing encephalopathy in an encephalopathic child.

Question: Thank you for your very interesting answers as well as your support. We look forward your plenary lecture in the forthcoming '5th workshop on Paediatric Virology' on radiology findings of RSV-positive bronchiolitis in children.

\section{Acknowledgements}

This article is published in the third supplement issue of the Experimental and Therapeutic Medicine, which is dedicated to Paediatric Virology. This edition is performed in the context of the '5th workshop on Paediatric Virology' (Sparta, Greece, October 12, 2019) organized by the Paediatric Virology Study Group (PVSG) and supported by the Department of Clinical Virology of the University of Crete School of Medicine and the First Department of Paediatrics of the University of Athens School of Medicine. We would like to thank Dr Georgia Papaioannou for this educational and inspirational interview-style article. We would also like to thank all the members of the PVSG for their interesting questions to Dr Papaioannou and their valuable comments.

\section{References}

1. Papaioannou G: Metallic stents. Application in the treatment of iliac artery atherosclerotic disease (PhD Thesis). Medical School of Athens, National and Kapodistrian University of Athens, Athens, 2004.

2. Papaioannou G: Imaging diagnosis and follow-up of congenital lung anomalies. In: Inherited and Congenital Lung disease, 3rd Paediatric Clinic of University of Athens, pp172-186, 2nd Volume, Athens, 2012 (In Greek).

3. Papaioannou G: Imaging Principles in Pediatric Oncology. In: Imaging in Clinical Oncology. Springer, Milano, 2013.

4. Papaioannou G: Lymphomas in Children and Adolescents. In: PET/CT in Lymphomas. A Case-Based Atlas. Springer International Publishing Switzerland, 2016.

5. Papaioannou G and McHugh K: Imaging Principles in Pediatric Oncology. In: Imaging in Clinical Oncology, Second Edition. pp63-69. Springer International Publishing, 2018.

6. Papaioannou G, Young C and Owens CM: Multidetector row CT for imaging the paediatric tracheobronchial tree. Pediatr Radiol 37: 515-529, quiz 612-613, 2007.

7. Papaioannou G, Sebire NJ and McHugh K: Imaging of the unusual pediatric 'blastomas'. Cancer Imaging 9: 1-11, 2009.

8. Papaioannou G, Koussidis G and Michala L: Magnetic resonance imaging visualization of a vaginal septum. Fertil Steril 96: 1193-1194, 2011.

9. Papaioannou G, Kambas I, Tsaoussoglou M Panaghiotopoulou-Gartagani P, Chrousos G and Kaditis AG: Age-dependent changes in the size of adenotonsillar tissue in childhood: Implications for sleep-disordered breathing. J Pediatr 162: 269-74.e4, 2013.

10. Michala L, Vlachopapadopoulou E, Tsimaris P, Papaioannou G, Paisiou A, Peristeri I, Michalacos S and Kitra V: Resolution of Hematocolpos in Adolescents Affected with Graft vs Host Disease. J Pediatr Adolesc Gynecol 31: 536-539, 2018.

11. Pastroma A, Papaioannou G, Raissaki M and Alexopoulou E: The common among the rare: A pictorial essay of the most common diffuse lung diseases in children. Can Assoc Radiol J 69: 468-478, 2018.

12. Brountzos EN, Papaioannou G, Alexopoulou E and Kelekis DA: Arteriovenous Malformation of the Foot: Management with Transcatheter Embolization with PVA and Alcohol. Case Club. January 2000. Available at: http://www.scvir.org.

13. Papaioannou G, Tavernaraki K and Kelekis DA: Myositis ossificans of the gluteal muscles. Case 3091. http://www.eurorad. org/case.php?id=3091. Accessed May 5, 2008.

14. Papaioannou G, Nassis N and Kelekis DA: Transitional cell carcinoma of the ureter demonstrated with computed-tomography. Case 3085. http://www.eurorad.org/case.php?id=3085. Accessed April 30, 2009.

15. Papaioannou G: Panorama HFO ideal for fetal and pediatric scans. NetForum Community, 2010.

16. Mammas IN, Theodoridou M, Kramvis A, Thiagarajan $\mathrm{P}$, Gardner S, Papaioannou G, Melidou A, Koutsaki M, Kostagianni G, Achtsidis V, et al: Paediatric Virology: A rapidly increasing educational challenge. Exp Ther Med 13: 364-377, 2017.

17. Mammas IN, Greenough A, Theodoridou M, Kramvis A, Rusan M, Melidou A, Korovessi P, Papaioannou G, Papatheodoropoulou A, Koutsaftiki C, et al: Paediatric Virology and its interaction between basic science and clinical practice (Review). Int J Mol Med 41: 1165-1176, 2018.

18. Mammas IN, Theodoridou M, Thiagarajan P, Melidou A, Papaioannou G,KorovessiP,Koutsaftiki C,Papatheodoropoulou A, Calachanis M, Dalianis T, et al: A paediatric influenza update 100 years after the Skyros island Spanish flu outbreak. Exp Ther Med 17: 4327-4336, 2019.

19. Papaioannou G: Multimodality imaging of viral infections in children: CNS and beyond. Int J Mol Med 38: S65, 2016.

20. Papaioannou G: Foetal and neonatal brain imaging in viral infections. Int J Mol Med 40: S39, 2017.

21. Papaioannou G: Radiology issues of influenza in children. Int $\mathbf{J}$ Mol Med 42: S18, 2018.

This work is licensed under a Creative Commons Attribution-NonCommercial-NoDerivatives 4.0 International (CC BY-NC-ND 4.0) License. 